\title{
Outdoor Logistics Tracking Solution Based on Computer Augmented Reality and RFID Hybrid Technology
}

\author{
KANG Jian ${ }^{1 a}$, ZHANG Peng ${ }^{2 b}$ \\ Department of Information and Technology Engineering, Tang Shan Vocational and Technical \\ College, Tangshan Hebei 063004, P.R.China
}

Keywords: augmented reality (AR), radio frequency identification (RFID), logistics, no marker tracking

\begin{abstract}
The logistics process improvement scheme is described in this paper using AR (augmented reality) and mixed RFID (radio frequency identification) technology. Now, the augmented reality and RFID technology are well known and widely applied in practice, they are merged together can enhance the visual function of the system. This paper provides the theoretical characteristics of the logistics of radio frequency identification, the augmented reality technology and relevant concepts and theoretical models are expressed. Model is described based on outdoor target tracking and visualization of three-dimensional model, and the solution of radio frequency identification technology is obtained. For the development and test of the test platform, the product provides the physical structure and basic operations and basic principle. Outdoor target tracking and 3D model visualization may significantly affect and improve the logistics process in different areas.
\end{abstract}

\section{Introduction}

RFID technology can identify a single piece of goods, but for a longer distance, RFID has a certain limitation, which is bound for the application of the technology. The development of hybrid RFID technology is helpful to realize the target tracking in outdoor environment, and it is helpful to determine the location of the target by using GPS. RFID can display an object identifier, but single identifier information may not be sufficient, because the analyst and the warehouse keeper will make mistakes if the goods are selected based on a single identifier.

AR technology can display additional data in an appropriate environment, for example, a 3D model is constructed based on text information, pictures, video frames, schema and real-world objects. AR is determined by the position of the object and the identifier, and it can display the virtual reality real-time object selection. The amplification process can provide additional data visual recognition of the current object, which helps to reduce the user's choice error in other target objects. Its fast content recognition function ensures the quick decision, reduces the time parameter value, it significantly improves the logistics process.

\section{Augmented reality technology and limiting factors}

AR is applied in the real world environment directly or indirectly, and it is supplemented by the visual entity formed by the special hardware, software and auxiliary elements. Augmented reality (AR) definition is described as: virtual information enhancement all activities in the real world environment as the main target, through the virtual information abundance human consciousness and ability. AR can link the virtual information to the real world. AR concept is actually using the virtual imagination to change the real environment. For example, the TV channel is playing sports translation, and the TV screen with the table shows the actual results of sports events or other non video basic information. This is the simple AR typical example, the captured video information is changed into interactive, digital control.

Because of the simple and successful testing based on the system AR tags, AR can be widely used in indoor personal computers and mobile devices. But there are some solutions in the outdoor environment, such as equipped with an internal sensor device, and it can be used in outdoor 
amplification process with positioning information. In turn, GPS technology can also be used in outdoor environments for location of direct links.

AR solutions are using a variety of techniques for data speculation and description. In the marker system mentioned above, using a readable, stable logo as identifier for amplified entity position. The user can see the camera video output device and the target of augmented reality in the real environment. Recognition of 3D model can use RFID solution, which can identify the object itself and the related 3D model. When completed the recognition, the system can display the 3D model tag. The solution also has its weakness. The camera must be very clear to see the mark and put a three-dimensional model in a limited angle. The problems in the process of eye identification marking are caused by glare, light angle, camera rotation, vibration, camera quality and other factors. In turn, a marker free system can identify the position of a point in a video frame or a sensor, such as a GPS. The visual elements are used to mark the position. The non marking system can also use the method above to provide more accurate position determination in the application of outdoor environment. To achieve such a solution, it is required to have a good performance, stable illumination conditions, perspective and scalability of the fast algorithm and data processing method. The main differences between the labeled and unlabeled solutions are the visual distance and the target location.

\section{Hybrid radio frequency identification system and non marking solution}

Accounting, tracking and storage of computer systems are very useful, they are necessary in obtaining movement and location information of objects. This information has great significance, and it is necessary to almost any system that tracks the flow of a target. Next, the author will discuss hybrid integration of radio frequency identification technology and global positioning technology, and try to describe integrated amplification reality solutions. In the outdoor environment, the task of determining the location is completed, the global positioning system the most appropriate and cost-effective technology. However, the process of determining the location is restricted in several cases, most of the time, it is relevant to sensors and low earth orbit satellite receiving signal visibility.

\subsection{Architecture of hybrid video recognition system}

Most hybrid video recognition solutions use active RIFD tags. Activity RIFD tags can provide faster data exchange with higher capacity. These systems use LEO satellite for tracking to identify various targets, such as containers, cars, construction parts, animals and so on. System structure of typical RFID technology is constructed based on the incorporation of global positioning system components. The operation of the global positioning system is established based on GPS sensor readings. The Savi technology activity RFID tags need to be activated several times a day, and then send it to the nearest LEO satellite through the data channel to determine the latitude and longitude of the global positioning system. Either as an active RFID tag or a satellite tag, hardware equipment will send its unique identification number.

When the tag is located in a supply chain with a radio frequency identification reader, the tag automatically closes the satellite controller. The tag is smart enough to know when the container is out of position, and when it is not in the range of the radio frequency identification reader, it can automatically open the satellite controller. However, each label and satellite systems are required to communicate the need to pay. On the other terminal, it must taken based on network applications, which is easy to use for end users, but more importantly, the application is cross platform compatible, so it doesn't matter what users are using desktop computers, Android tablets, iPhone or other devices. The structure of remote RIFD tag identification is shown in Figure 1.

As shown in Figure 1, the modern logistics hardware units are characterized by other sensors that can track the target's temperature and the lack of local motion information by satellite data channel or multi band mobile network. 


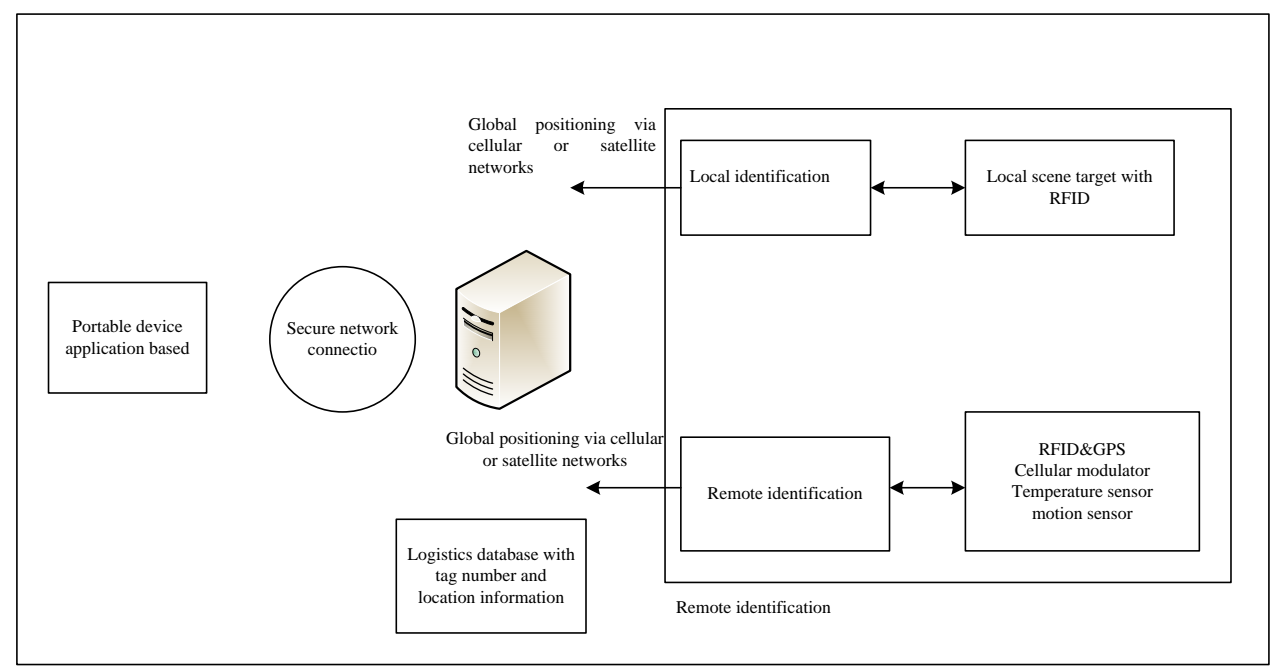

Fig.1 Structure of remote RIFD tag identification

Analysis of modern logistics system, tracking target is not only important for the global scope. The solution can be increasingly popular in a short distance tracking object. If the general warehouse object is registered from one place to another, or the status of the object is changed, the modern system will provide the RTLS (real-time positioning system) to track the visual real-time object in the indoor environment (see Figure 1). The level of detail is very accurate, so that you can see the remote real-time animation, see how the goods are removed from the shelves of the warehouse workers, how to place the cart, how to flow in the warehouse.

\subsection{Implementation and best example of hybrid radio frequency identification system}

A lot of examples show that the use of hybrid radio frequency identification technology is more and more common. The remote tracking scheme is implemented and managed by the Impeva laboratory, it uses a device that is similar to the RFID tag, and the technology is used for satellite communications. Cellular communications are helping to ensure that other national troops are receiving the necessary supplies. The solution uses Impeva device called the global tag unit (GSU), which is installed on the truck as an active RFID tag, it can send a unique serial number and GPS data to determine the vehicle and its location. However, GSU does not communicate with the RFID reader, but it uses the global satellite and multi band cellular network to transmit data. GSU can also store a list of trucks, it is powered by batteries, including GPS receivers and satellite and cellular modems. The unit can customize the geographic area that serves more than 40 users, and can be set up to start the communication when the GSU is not in the area. Equipment can also be queried any time. GSU sends their data to the management center of the global mark device at the Impeva laboratory data center in the United States. The server runs based on Web software, to process and store all the information collected by GSU, and finally get the remote user unit. All relevant cargo data and continuous GPS positions are reported and encrypted prior to passing through satellite or cellular data channels. The US military can use Google maps to create an agile web-based application to secure access to the information. The military can determine the location of the vehicle by entering the serial number of a particular vehicle, and it can be described by a retrospective description of the goods contained in the vehicle.

SIEMENS information technology solutions and services is another successful case. It is proved that the technology based on radio frequency identification equipment can be used to monitor the testing of shipping containers at sea. Tests show that the technology can close the market which can buy only available on land RIFD data cargo monitoring RFID system gap. It continues to monitor the flow of containers from the port to the final destination. In order to develop this system, SIEMENS information technology solutions and services builds cooperation with the SIEMENS NOKIA network service, the network service provides a solution based on GSM communications, and that is a smaller version of the cellular network.

The international Oil and Natural Gas Corp BP created the RFID and GPS systems for each item of shipping cargo. Shipping starts in Europe flows to South Korea eventually. The system uses the 
economic and Planning Commission 2 RFID tags, which are loaded on each of the goods which attached to the tag, signed 2D bar codes basically. Using a mobile reader can interpret these bar codes. Using bar code in the system is not the only target recognition method. The cargo on board can be placed in different ways, such as a long string of small boxes. Each object in the system is to use passive and active RFID tags. The tag is equipped with a GPS transmitter for measuring the position of the goods, and the measurement accuracy can be accurate to 1 meters. Ultimately, users can receive the map of the world cargo location image information as well as the description of the warehouse cargo information.

The above mentioned system is a few successful cases that have recognized the potential of radio frequency identification and global positioning. In turn, this paper describes the theory of the AR system with additional features. In fact, the contents of AR identifies can be displayed on the mobile device, mobile phone, tablet PC or HMD (head mounted display) on the screen. Users (warehouse operators or customers) can choose the appropriate container or other packaging by observing the 3D model of the selected product. This type of system also requires real-time GPS data and display the correct model projection technique on real video frames.

\subsection{Combination of augmented reality and RF technology}

Hybrid radio frequency technology system has high precision. If the system is designed to be as accurate as possible, the system can significantly enhance the target control, improve the equipment usage and reduce the cost. In order to carefully design the installation and control system, the developer must pay attention to the problem of data duplication, data delivery, RFID tag installation and other components.

If the system receives the GPS data, it can determine the location of RFID tag. If the system knows the location of RFID tag, you can use the same location information to calculate the two-dimensional or three-dimensional data in the virtual space or real space, to achieve the appropriate amplification of the coordinate position. As mentioned before, two data communication channels can be used to transmit radio frequency identification information: one is via satellite or a cellular network, the other is a radio frequency identification reader. When the system receives the necessary data, the product reaches the user's perspective, the physical products can be read by mobile phones, tablet PCs or HMD (head mounted display), or uses the virtual information to replace.

Correctly designing the virtual elements, only the latitude and longitude data are not enough. It also needs to calculate the position and direction of the observer. In this case, the camera is referred as environmental observer, it can capture real-time video and integrate the position sensors, the interaction between the object and the observer is tracked, and the object and the observer can be static, also can exercise.

Hybrid tracking is a method for real-time detection of GPS location data and camera location. GPS accuracy is an important issue that can be increased with the differential global positioning system, the accuracy of reading is set in 1-3 meters, the differential global positioning system can be tracked, and the ordinary GPS is set in the 4-20. GPS receivers receive differential GPS using fixed networks. The error factor is calculated between the predefined position and the position of the satellite signal. This error is transmitted as an FM signal to the local GPS receiver, making the necessary corrections to the reading.

\section{Applied research}

Logistics industry sector to a certain extent affect all parts of the traditional industries, and almost impossible to get without a few supply chain of consumer products. Logistics involves various parts of the world from one end to the other end, starting with the transport of machinery parts, food or clothing, and a small portion of the aggregate production line. Such an opportunity creates more and more complex transport network, so that the logistics translates into a rapid expansion of the global industry. If the workers are placed in the middle of the warehouse logistics chain, they may not be able to endure the long time constraints, thus causing the system to be the bottleneck. Errors can sometimes produce very high costs, for example, that an error has been 
installed in a previously scheduled car, or has been sent to the other side of the planet. For this reason, Workers are trapped in a high level of work pressure, and many of the systems have to be reduced to their workload by working in a more efficient way to reduce the errors.

Today, different techniques are used to prepare the information for order selection. But almost all have disadvantages. For different techniques, the error rate is between $0.1 \% \sim 0.8 \%$. This means that there are 1 to 8 orders within 1000 is wrong. And there are many kinds of mistakes, such as the wrong goods or quantity. Customers usually do not accept even an error within 1000 (e.g., the original equipment manufacturer), because each error can lead to a stop of the production line. AR system may reduce the pressure of the workers, and reduce the error.

Figure 2 shows the basic flow chart of the AR-RFID system, which is constructed based on the selection of appropriate entities in the database. Based on the GPS location data, the coordinates of the target are calculated. Before this, the user is located close enough to the target. If the GPS signal is too weak to cause the position information to be transmitted, the other non marking method can be used on the basis of the video frame identification. Regardless of the choice of what kind of label free method, the core idea is the collection of now different types of AR methods, the development of a universal outdoor AR projection solution is obtained.

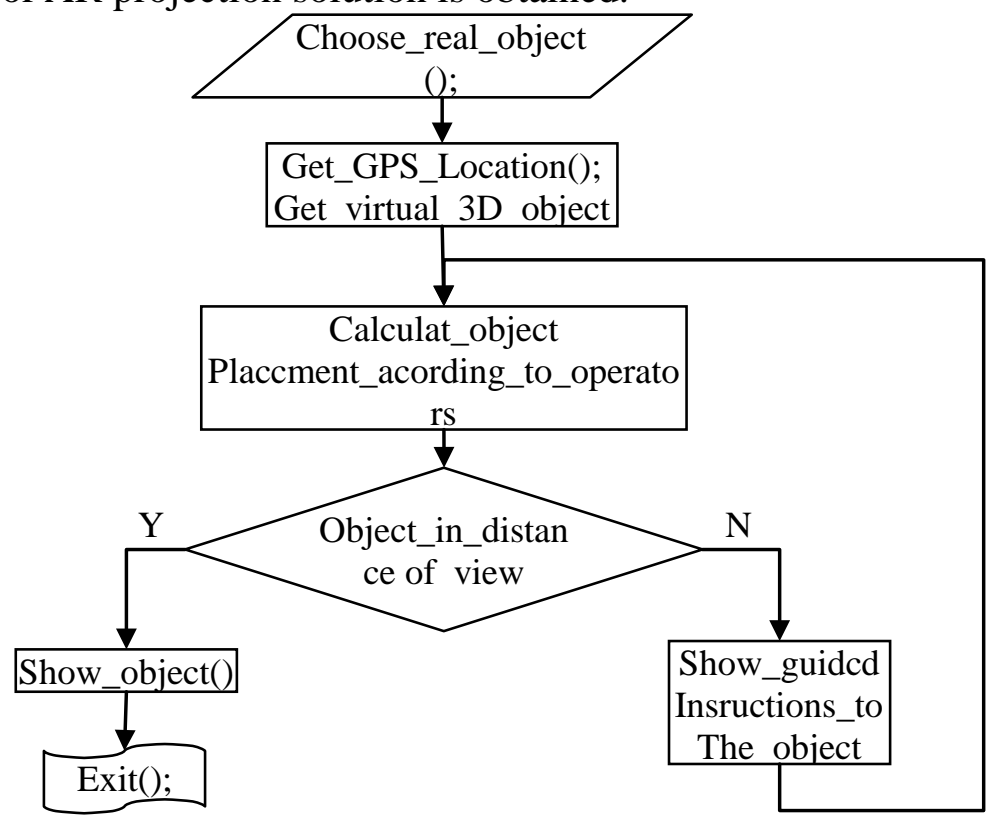

Fig. 2 Basic flow chart of the AR-RFID system

In the system, an active RFID tag must be used. These tags can contain more data in memory, providing faster data flow. Activity tags can work at any frequency, and can be processed by passive or semi passive tags for faster data flow and information capacity. Just as the SIEMENS information technology solutions (It is shown in Figure 3), RFID service and GPS system are constructed. System allows increase the safety level of port and shipping services. Many have been done on the identification and registration services, but also through the observation of the important elements of the logistics process to do more, because not all employees are familiar with the logistics clues, especially for the new employees. 


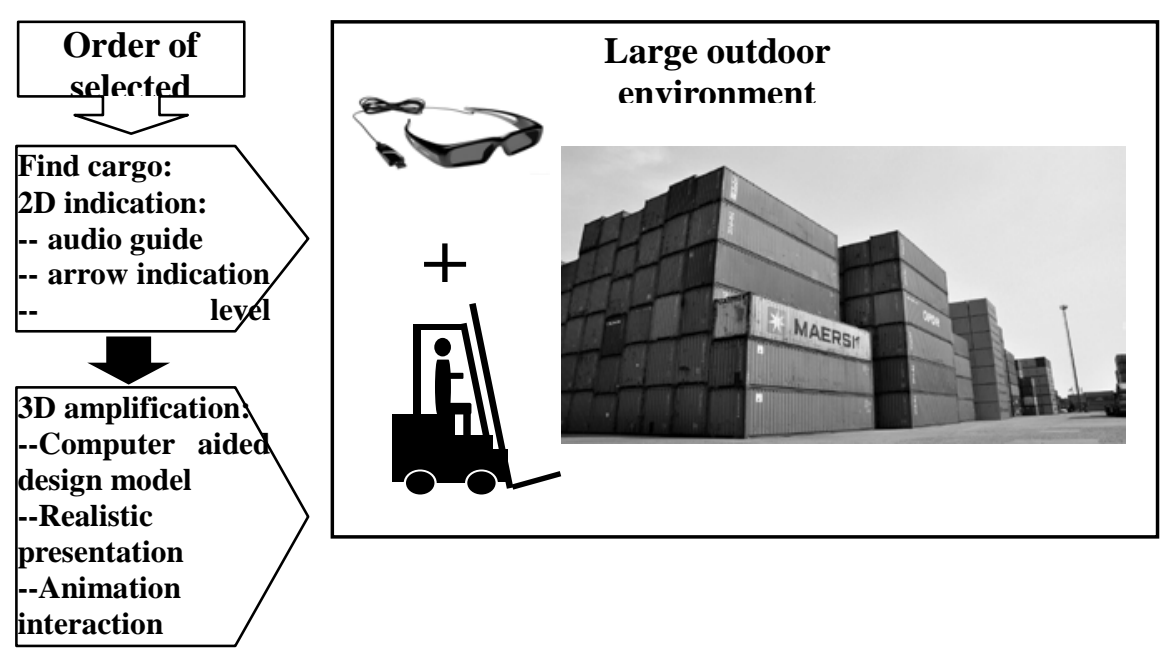

Fig.3 Improved target search mode

\section{Conclusion}

Logistics plays a very important role in the business and trade of today's world. Logistics has a wealth of history, almost all areas of economics can be used in various fields of economics. At the same time, a wide range of technologies are combined with the logistics technology, including logistics and related processes, it must be developed to gain promotion. As Ginters had researched in the field of logistic before, logistics can be combined in different technologies, such as radio frequency identification and augmented reality. The main benefit is to identification, detection and identification of products or other warehouse goods. By improving these processes, the logistics system can reduce picking errors. Because the requirements of augmented reality can enhance the correctness of choice, the main responsibility of AR is to display additional data on specific items to the user. Through screen or HMD, the worker can always correctly identify the product. The previous results have been implemented in an indoor environment, thanks to the idea, which has an important role in the implementation of the outdoor environment. In order to realize this technology in outdoor environment, the augmented reality no marker technology and hybrid radio frequency identification technology are used in combination. Developers should pay attention to the global positioning system signal strength, radio frequency identification tag location and the function of the mobile device. Hybrid radio frequency recognition and augmented reality without marking system in outdoor environment logistics process can provide additional level of security, and it is easier for target recognition, the goal recognition and content display and system flow of goods can be obtained more easily, it has good application value in practice.

\section{References}

[1]. ZHENG Zhen,WANG Liyuan,ZHOU Yong. Chaos Theory and Its Application in Ships Target Recognition[J].Ship Electronic Engineering,2013,33(5): 48-50.

[2]. Li Yang-qun, Shen Su-bin, and Xu Bin. Technology of web of things: asurvey[J]. Journal of Nanjing University of Posts and Telecommunications (Natural Science), 2014, 34(2): 32-42.

[3]. Benedikt O, Kay R, Friedemann M, et al.. A real-time search engine for the web of things[C]. Internet of Things, Tokyo, 2010: 1-8.

[4]. Li Zeng-ke, Gao Jing-xiang, Wang Jian, et al.. Inerialdynamic model of GPS/INS integrated navigation based on newton interpolaion[J]. Geomatics and Information Science of Wuhan University, 2014, 39(5): 591-595.

[5]. Shang Jun-liang and Fang Min. New optimized method of high-precision grey GM(1,1) forecasting model[J]. Journal of Electronics \& Information Technology, 2010, 32(6): 1301-1305. 
